\title{
SPATIAL DISTRIBUTION OF BACK SCATTERING IN THE NUCLEAR AREA OF THE NON-CATARACTOUS HUMAN LENS
}

\author{
WEN QIAN, PER SÖDERBERG, BO LINDSTRÖM, ENPING CHEN, KARIN MAGNIUS and BO \\ PHILIPSON \\ Stockholm, Sweden
}

\begin{abstract}
SUMMARY
The spatial distribution of back scattering from a vertical cross-section of the nuclear area in non-cataractous human lens was investigated. The cross-section was centred on the pupil. There is no significant difference in average nuclear back scattering between sides. The distribution of back scattering along an axis perpendicular to the anatomical axis can be modelled as a second-order polynomial. It is believed that the increase of back scattering in the central region corresponds to the peak protein concentration in the centre of the nucleus. This information was obtained by imaging back scattering with Scheimpflug photography. The back scattering was measured photometrically in a $3 \times 10$ matrix of measuring areas within the lens nucleus. Each measuring area was $0.20 \times 0.20 \mathrm{~mm}$ in size. It is anticipated that the established model will allow detection of nuclear cataract with high sensitivity, especially in early stages.
\end{abstract}

Scheimpflug photography has proved to be an important tool for quantitative measurements of lens nuclear transparency. ${ }^{1-10}$

Nuclear cataract develops symmetrically around the anterior-posterior axis." Therefore, one Scheimpflug photographic section is sufficient to represent the opacity in the nucleus. " On the other hand, cortical cataract is always highly asymmetrical, ${ }^{12}$ so that even several photographic sections might not be sufficient for detecting a change in opacity in the cortex. ${ }^{12}$ Therefore, Scheimpflug photography is a suitable technique for objective documentation of nuclear cataract but not cortical cataract. ${ }^{13}$

In principle, to detect any change in nuclear back scattering an area as large as possible in the nucleus should be measured, and the cortical region should be excluded to

From: Research Laboratory, St Erik's Eye Hospital, and Department of Medical Biophysics, Karolinska Institute, Stockholm, Sweden.

Correspondence to: Wen Qian, Research Laboratory, St Erik's Eye Hospital, S-112 82 Stockholm, Sweden. obtain a high precision. ${ }^{11.12}$ However, a variation in location and size of the nucleus among individuals is expected and the border between the nucleus and the cortex is not always well defined. To overcome these problems and thus to optimise the possibility of detecting a change in the nuclear density, a common lens nuclear area was established. ${ }^{14}$

If there is a characteristic distribution of back scattering in the common lens nuclear area that can be determined with high precision, the sensitivity for detection of changes in back scattering increases if the characteristic distribution is considered.

The purpose of the present study was to estimate the physiological spatial distribution of back scattering in the nuclear area. Further, it was intended to analyse how a change in back scattering from the lens nucleus can be measured with adequate sensitivity.

\section{MATERIALS AND METHODS}

The distribution of back scattering in the nucleus of clinically non-cataractous human lenses was analysed with photometry of Scheimpflug photographs.

\section{Experimental Design}

Subjects were randomly selected from a control group of a continuing clinical study testing a cholesterol-decreasing drug. All subjects had clear lenses at slit lamp examination and a visual acuity of at least $1.0(6 / 6)$.

In total, Scheimpflug photographs from 10 individuals ( 8 males and 2 females, aged 52-72 years) were measured. Several photographs were taken on the same occasion for each eye of each subject. Two randomly selected photographs from each eye were analysed. In each photograph a $3 \times 10$ matrix of areas in the nucleus was measured (Fig. 1).

\section{Lens Photography and Densitometry of the Photographs}

The lens was photographed with a Topcon SL-45 slit lamp 
camera. The camera has a built-in reference system, consisting of a five-step grey scale reference system. All photographs were taken at vertical slit orientation $\left(90^{\circ}\right)$. The slit light was projected along the sagittal plane towards the lens. The film (Kodak TX 5063) was developed according to a standard method. ${ }^{2}$

The spatial distribution of back scattering in the nucleus was measured with a modified Leitz microscope photometer $^{15}$ equipped with a recording device that allowed two-dimensional positioning of the measuring area with a micrometer resolution. The measuring area was $0.2 \times 0.2 \mathrm{~mm}$ in the object plane, and the linear magnification was 30 times.

\section{Measurement Procedure}

The photograph with the lens image was placed on the microscope stage. A virtual $X-Y$ coordinate system was established over the lens photograph oriented so that the $\mathrm{X}$-axis was parallel with the tangent to the anterior vertex of the lens and the Y-axis coincided with the anatomical axis of the lens (Fig. 1). Each of a $3 \times 10$ matrix of areas (Fig. 1) in each photograph was measured photometrically. The total area measured was selected on the basis of the common lens nuclear area. ${ }^{14}$ The photometric reading and its corresponding position of each area were recorded. Each photometric measurement was converted to a corresponding grey scale level by calibration of the image reading to the grey scale reference on the same photograph. ${ }^{16}$

\section{Data Analysis}

The data were analysed according to a statistical model utilising an analysis of variance (Appendix 1).

Further, the experiment was planned to allow polynomial regression with orthogonal comparisons for determination of a possible characteristic spatial distribution. ${ }^{18}$

\section{Statistical Parameters}

Considering the small sample size, the significance level and the confidence coefficient were set to 0.05 and 0.95 , respectively.

\section{RESULTS}

The analysis of the variation of back scattering within the nuclear area of the 20 lenses is presented in Table I.

\section{Random Sources of Variation}

The estimated variance for individuals $\left(\sigma_{A}^{2}\right)$ was $1230 \times 10^{-4}$ (grey scale value). ${ }^{2}$ This was obtained on the basis of the expressions of the expected mean square for individuals and photographs (Table I).

The estimated variance for photographs $\left(\sigma_{\mathrm{C}}^{2}\right)$ was $127 \times 10^{-4}$ (grey scale value). ${ }^{2}$ This was obtained from the expressions of the expected mean square for photographs and measurement error (Table I). Here, the measurement error $\left(\sigma_{\varepsilon}^{2}\right)$ was approximated as $15 \times 10^{-4}$ (grey scale value $)^{2}$ neglecting the variance for interaction among photographs, $X$-locations and $Y$-locations, $\sigma_{C \zeta \eta}^{2}$ (Table I).
As measurement error $\left(\sigma_{\varepsilon}^{2}\right)$ was $15 \times 10^{-4}$ (grey scale value $)^{2}$, the coefficient of variation for measurements was about $3 \%$.

\section{Variation Between Sides}

There was no significant difference in back scattering between the right and the left eye (Table I: Sides. $F_{1: 9}=0.73<F_{1 ; 9 ; 0.95}=5.12$ ).

\section{Spatial Distribution of Back Scattering}

There is a variation of back scattering along the $X$-axis (Table I: $X$-locations). There is no significant variation of back scattering along the $Y$-axis (Table I: $Y$-locations). There is no significant difference between the right and left eye considering the distribution of back scattering along the $X$-axis and $Y$-axis, respectively (Table I: Int. $(\mathrm{S}-\mathrm{X})$, Int. $(\mathrm{S}-\mathrm{Y}))$.

There is a two-dimensional variation of back scattering along the $X$ - and $Y$-axes (Table I: Int. $(\mathrm{X}-\mathrm{Y})$ ). There is no significant difference in the two-dimensional distribution of back scattering between sides (Table I: Int. (S-X-Y)).

Since the variation along the $X$-axis was found to be significant, the distribution of back scattering along the $X$-axis was further analysed using orthogonal comparisons. $^{18}$

\section{Pattern of the Back Scattering}

The orthogonal comparisons (Table II) showed that the distribution of back scattering along the $X$-axis can be represented by a second-order polynomial (equation 1).

$$
Z=a_{0}+a_{1} X+a_{2} X^{2}
$$

where $Z$ is the grey scale value, $X$ the position along the $X$-axis, and $a_{0}, a_{1}$ and $a_{2}$ are regression coefficients, specific for each photograph.

The data for each photograph were fitted to a model corresponding to equation (1) with polynomial regression.

\section{Estimate of Regression Parameters in a Population}

The sources of variation in estimating these regression parameters in a population were analysed with an analysis of variance according to Appendix 2-1. The result is given in Table III. Here, the variation between sides was considered as a random factor since the difference between sides and the interaction between individuals and sides are not significant (Table I: Sides, Int.(I-S)).

A $95 \%$ confidence interval for $a_{0}, a_{1}$ and $a_{2}$, respectively, was then estimated (Table IV) according to Appendix 2-2 using the mean square for individuals in Table III. The estimated physiological spatial back scattering along the $X$-axis is plotted by substituting the means of $a_{0}, a_{1}$ and $a_{2}$ into equation (1) (Fig. 2).

\section{Sensitivity of the Method}

The sensitivity of the method was estimated by comparing the coefficients $\left(a_{0}, a_{1}\right.$ and $\left.a_{2}\right)$ derived from the original grey scale data with those derived from simulated grey scale data. 
Table I. Analysis of variance of back scattering within the nuclear area in the non-cataractous lens

\begin{tabular}{|c|c|c|c|c|}
\hline $\begin{array}{l}\text { Source of } \\
\text { variation }\end{array}$ & $\begin{array}{c}\begin{array}{c}\text { Degrees of } \\
\text { freedom }\end{array} \\
\end{array}$ & $\begin{array}{l}\text { Mean square } \\
\text { (grey scale) }^{2}\end{array}$ & Expected mean square & $\begin{array}{c}\text { Test statistic } \\
\left(F_{\mathrm{fl}: \mathrm{f2} ; 0.095}\right)\end{array}$ \\
\hline Individuals (I) & 9 & 15.1491 & $\sigma_{\epsilon}^{2}+d n \sigma_{\mathrm{C}}^{2}+b c d n \sigma_{\mathrm{A}}^{2}$ & \\
\hline Sides $(\mathrm{S})$ & 1 & 0.4960 & $\sigma_{\epsilon}^{2}+d n \sigma_{\mathrm{C}}^{2}+c d n \sigma_{A \beta}^{2}+a c d n \kappa_{\beta}^{2}$ & $0.73(5.12)$ \\
\hline Photographs (P) & 20 & 0.3816 & $\sigma_{\epsilon}^{2}+d n \sigma_{\mathrm{C}}^{2}$ & \\
\hline$X$-locations $(\mathrm{X})$ & 9 & 0.5681 & $\sigma_{\epsilon}^{2}+n \sigma_{C \zeta}^{2}+b c n \sigma_{A \zeta}^{2}+a b c n \kappa_{\zeta}^{2}$ & $27.44(1.99)$ \\
\hline$Y$-locations $(\mathrm{Y})$ & 2 & 0.5725 & $\sigma_{\epsilon}^{2}+d \sigma_{C \eta}^{2}+b c d \sigma_{A \eta}^{2}+a b c d \kappa_{\eta}^{2}$ & $1.29(3.55)$ \\
\hline Int. (I-S) & 9 & 0.6774 & $\sigma_{\epsilon}^{2}+d n \sigma_{C}^{2}+c d n \sigma_{A \beta}^{2}$ & $1.78(2.40)$ \\
\hline Int. $(\mathrm{I}-\mathrm{X})$ & 81 & 0.0207 & $\sigma_{\epsilon}^{2}+n \sigma_{C \zeta}^{2}+b c n \sigma_{A \zeta}^{2}$ & $2.44(1.35)$ \\
\hline Int. (I-Y) & 18 & 0.4446 & $\sigma_{\epsilon}^{2}+d \sigma_{C \eta}^{2}+b c d \sigma_{A \zeta}^{2}$ & $15.07(1.87)$ \\
\hline Int. $(S-X)$ & 9 & 0.0091 & $\sigma_{\epsilon}^{2}+n \sigma_{C \zeta}^{2}+c n \sigma_{A \beta \zeta}^{2}+a c n \kappa_{\beta \zeta}^{2}$ & $0.92(1.99)$ \\
\hline Int. (S-Y) & 2 & 0.0090 & $\sigma_{\epsilon}^{2}+d \sigma_{C \eta}^{2}+c d \sigma_{A \beta \eta}^{2}+a c d \kappa_{\beta \eta}^{2}$ & $0.18(3.55)$ \\
\hline Int. $(\mathrm{P}-\mathrm{X})$ & 180 & 0.0085 & $\sigma_{\epsilon}^{2}+n \sigma_{C \zeta}^{2}$ & \\
\hline Int. (P-Y) & 40 & 0.0295 & $\sigma_{\epsilon}^{2}+d \sigma_{C \eta}^{2}$ & \\
\hline Int. $(X-Y)$ & 18 & 0.0179 & $\sigma_{\epsilon}^{2}+\sigma_{C \zeta \eta}^{2}+b c \sigma_{A \zeta \eta \eta}^{2}+a b c \kappa_{\epsilon \eta}^{2}$ & $2.06(1.67)$ \\
\hline Int. $(\mathrm{I}-\mathrm{S}-\mathrm{X})$ & 81 & 0.0099 & $\sigma_{\epsilon}^{2}+n \sigma_{C \zeta}^{2}+c n \sigma_{A \beta \zeta}^{2}$ & $1.17(1.35)$ \\
\hline Int. $(\mathrm{I}-\mathrm{S}-\mathrm{Y})$ & 18 & 0.0512 & $\sigma_{\epsilon}^{2}+d \sigma_{C \eta}^{2}+c d \sigma_{A \beta \eta}^{2}$ & $1.74(1.87)$ \\
\hline Int. $(\mathrm{I}-\mathrm{X}-\mathrm{Y})$ & 162 & 0.0087 & $\sigma_{\epsilon}^{2}+\sigma_{C \zeta \eta}^{2}+b c \sigma_{A \zeta \eta}^{2}$ & $5.80(1.25)$ \\
\hline Int. $(S-X-Y)$ & 18 & 0.0041 & $\sigma_{\epsilon}^{2}+\sigma_{\zeta \zeta \eta}^{2}+c \sigma_{A \beta \zeta \eta}^{2}+a c \cdot \kappa_{\beta \zeta \eta}^{2}$ & $0.98(1.67)$ \\
\hline Int. (I-S-X-Y) & 162 & 0.0042 & $\sigma_{\epsilon}^{2}+\sigma_{C \zeta \eta}^{2}+c \sigma_{A \beta \zeta \eta}^{2}$ & $2.80(1.25)$ \\
\hline Int. $(\mathrm{P}-\mathrm{X}-\mathrm{Y})$ and error & 360 & 0.0015 & $\sigma_{\epsilon}^{2}+\sigma_{C \zeta \eta}^{2}$ & \\
\hline
\end{tabular}

Here, Int. is the abbreviation for the interaction factor. $a$, the number of individuals $(=10) ; b$, the number of sides ( $=2$ : right and left eye); $c$, the number of photographs in each eye in each individual $(=2) ; d$, the number of locations along the $X$-axis $(=10) ; n$, the number of locations along the $Y$-axis $(=3)$, $\sigma^{2}$, the expected variance for the indexed-random term: A, individuals; C, photographs; $\epsilon$, error. $K^{2}$, the factor corresponding to the variance for the indexed-fixed source: $\beta$, sides (right and left eye); $\zeta$, locations along the $X$-axis; $\eta$, locations along the $Y$-axis.

Table II. Orthogonal comparisons analysing the pattern of back scattering along the $X$-axis in the non-cataractous nuclear area

\begin{tabular}{|c|c|c|c|c|c|}
\hline $\begin{array}{l}\text { Source of } \\
\text { variation }\end{array}$ & $\begin{array}{c}\begin{array}{c}\text { Degrees of } \\
\text { freedom }\end{array} \\
\end{array}$ & $\begin{array}{c}\text { Sum of squares } \\
\text { (grey scale) }^{2}\end{array}$ & $\begin{array}{l}\text { Mean square } \\
\text { (grey scale) }^{2}\end{array}$ & Expected mean square & $\begin{array}{c}\text { Test statistic } \\
\left(F_{\mathrm{fl}: \mathrm{t} 2: 0.95}\right)\end{array}$ \\
\hline$X$-locations $(\mathrm{X})$ & 9 & 5.1129 & 0.5681 & $\sigma_{c}^{2}+n \sigma_{C \zeta}^{2}+b c n \sigma_{A \zeta}^{2}+a b c n \kappa_{\zeta}^{2}$ & $27.44(1.99)$ \\
\hline Linear & 1 & $* 0.6726$ & 0.6726 & $\sigma_{\epsilon}^{2}+n \sigma_{C \zeta}^{2}+b c n \sigma_{A \zeta}^{2}+\Delta_{1}$ & $32.49(3.96)$ \\
\hline Quadratic & 1 & $* 4.3601$ & 4.3601 & $\sigma_{c}^{2}+n \sigma_{C \zeta}^{2 \zeta}+b c n \sigma_{A \zeta}^{2}+\Delta_{2}$ & $210.63(3.96)$ \\
\hline Cubic & 1 & $* 0.0172$ & 0.0172 & $\sigma_{\epsilon}^{2}+n \sigma_{C \zeta}^{2}+b c n \sigma_{A \zeta}^{2}+\Delta_{3}$ & $0.83(3.96)$ \\
\hline Fourth & 1 & $* 0.0334$ & 0.0334 & $\sigma_{\mathrm{\epsilon}}^{2}+n \sigma_{C \zeta}^{2}+b c n \sigma_{A \zeta}^{2}+\Delta_{4}$ & $1.61(3.96)$ \\
\hline$>$ Fourth & 5 & $* 0.0296$ & 0.0094 & $\sigma_{\epsilon}^{2}+n \sigma_{C \zeta}^{2}+b c n \sigma_{A \zeta}^{2}+\Delta_{>t}$ & $0.45(2.33)$ \\
\hline Int $(\mathrm{I}-\mathrm{X})$ & 81 & 1.6767 & 0.0207 & $\sigma_{\epsilon}^{2}+n \sigma_{C \zeta}^{2}+b c n \sigma_{A \zeta}^{2}$ & \\
\hline
\end{tabular}

$X$-locations $(X)$ and Int $(\mathrm{I}-\mathrm{X})$ are from the Table I. Int. is the abbreviation for the interaction factor. The sum of the * values for comparisons is the sum of squares for $X$-locations. $a$, the number of individuals $(=10) ; b$, the number of sides $(=2$ : right and left eye $) ; c$, the number of photographs in each eye $(=2) ; n$, the number of locations along the $Y$-axis $(=3) . \sigma^{2}$, the expected variance for the indexed-random term; A, individuals, $\mathrm{C}$, photographs; $\epsilon$, error. $\kappa^{2}$, the factor corresponding to the variance of the indexed-fixed source; $\zeta$, locations along the $X$-axis. $\Delta_{4}$, a term for the contribution to the mean square from the $(q)$ th degree element $(q=1 \ldots 9)$.

Table III. Sources of variation for estimation of regression parameters

\begin{tabular}{lcrrrl}
\hline \multirow{2}{*}{$\begin{array}{l}\text { Sources of } \\
\text { variation }\end{array}$} & $\begin{array}{c}\text { Degrees of } \\
\text { freedom }\end{array}$ & \multicolumn{1}{c}{$a_{0}$} & \multicolumn{1}{c}{$a_{1}$} & \multicolumn{1}{c}{$a_{2}$} & \multicolumn{1}{c}{$\begin{array}{c}\text { Expected mean } \\
\text { square }\end{array}$} \\
\cline { 3 - 6 } Individuals & 9 & 5356 & 104 & 186 & $\sigma_{\epsilon}^{2}+n \sigma_{B}^{2}+b n \sigma_{A}^{2}$ \\
Sides & 10 & 208 & 39 & 85 & $\sigma_{\epsilon}^{2}+n \sigma_{B}^{2}$ \\
Photographs & 20 & 176 & 56 & 48 & $\sigma_{\epsilon}^{2}$ \\
\hline
\end{tabular}

$a_{0}$ (grey scale steps), $a_{1}$ (grey scale steps $/ \mathrm{mm}$ ) and $a_{2}$ (grey scale steps $\mathrm{mm}^{2}$ ) are regression parameters. $\sigma^{2}$ is the expected variance for the indexed source. A, individuals; B, sides; $\epsilon$, photographs + error. $b$, number of sides $(=2) ; n$, number of photographs $(=2)$.

When establishing the polynomial for each photograph, the back scattering was averaged over the three $Y$-locations at each of the ten $X$-locations (Fig. 1). These ten data points were used to establish a polynomial regression. Then the original data were altered in five different patterns (Fig. 3).
For each pattern, a polynomial was established for each photograph according to equation (1). The difference between the original coefficients and those after alteration were calculated for each regression coefficient on each photograph. The confidence interval for the difference of $a_{0}, a_{1}$ and $a_{2}$ was calculated respectively (Table $\mathrm{V}$ ) according to the same statistical model as in Appendix 2-1 and the same method as that used in Table IV.

\section{DISCUSSION}

In our previous work ${ }^{14}$ we defined a common lens nuclear area (CNA) for photometry of nuclear back scattering in Scheimpflug photographs. In the present study the largest rectangle $(0.68 \times 1.98 \mathrm{~mm})$ within the common lens nuclear area was calculated. The measuring area used in this study was set to a square of $0.20 \times 0.20 \mathrm{~mm}$. This size was selected to allow a sufficient number of points for a possible subsequent polynomial regression. Considering 
Table IV. The variance and the confidence interval for the regression coefficients

\begin{tabular}{lccc}
\hline $\begin{array}{l}\text { Coefficients of } \\
\text { polynomial }\end{array}$ & $\begin{array}{c}\text { Degrees of } \\
\text { freedom }\end{array}$ & $\begin{array}{c}\text { Mean square for } \\
\text { individuals }\left(10^{-4}\right)\end{array}$ & $\begin{array}{c}95 \% \text { confidence } \\
\text { interval }\end{array}$ \\
\hline$a_{0}$ (grey scale steps) & 9 & 5356 & {$[1.683 \pm 0.262]$} \\
$a_{1}$ (grey scale steps $\left./ \mathrm{mm}^{2}\right)$ & 9 & 104 & {$[0.041 \pm 0.037]$} \\
$a_{2}$ (grey scale steps $\left./ \mathrm{mm}^{2}\right)$ & 9 & 186 & {$[-0.208 \pm 0.049]$} \\
\hline
\end{tabular}

Mean squares for individuals are from Table III.

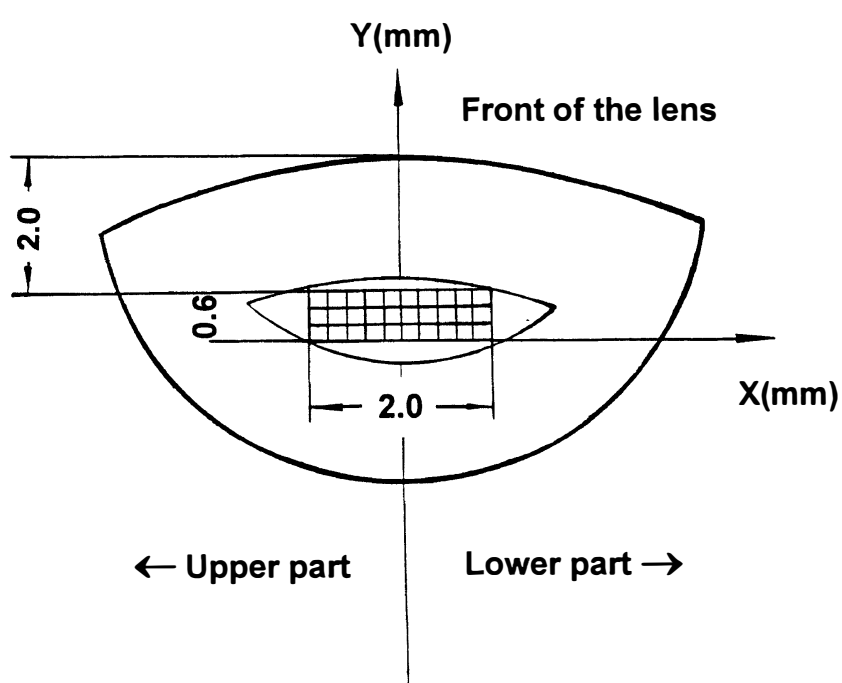

\section{Anatomical axis of the lens}

Fig. 1. Schematic drawing of a Scheimpflug image of the lens. A $3 \times 10$ matrix of measuring areas $(0.20 \times 0.20 \mathrm{~mm})$ was used for photometry. The area enclosing the matrix is the common lens nuclear area. ${ }^{14}$

Table V. Changes in the regression coefficients caused by increase of the back scattering in different patterns

\begin{tabular}{lccc}
\hline & \multicolumn{3}{c}{$95 \%$ confidence interval } \\
\cline { 2 - 5 } Pattern & $\begin{array}{c}\Delta a_{0} \\
(\mathrm{GS})\end{array}$ & $\begin{array}{c}\Delta a_{1} \\
(\mathrm{GS} / \mathrm{mm})\end{array}$ & $\begin{array}{c}\Delta a_{2} \\
\left(\mathrm{GS} / \mathrm{mm}^{2}\right)\end{array}$ \\
\hline Pattern 1 & $0.108 \pm 0.017$ & $0.000 \pm 0$ & $-0.175 \pm 0.028$ \\
Pattern 2 & $0.161 \pm 0.025$ & $0.001 \pm 0$ & $-0.238 \pm 0.037$ \\
Pattern 3 & $0.189 \pm 0.030$ & $0.002 \pm 0$ & $-0.223 \pm 0.035$ \\
Pattern 4 & $0.182 \pm 0.028$ & $0.003 \pm 0$ & $-0.107 \pm 0.016$ \\
Pattern 5 & $0.168 \pm 0.026$ & $0.004 \pm 0$ & $-0.021 \pm 0.005$ \\
\hline
\end{tabular}

$\Delta a$ is the difference between the coefficient derived from the altered grey scale level and that from the original. GS, grey scale steps. Five different patterns (Fig. 3).

the size of measuring area, and to facilitate the measure ment, the largest rectangle in the common nuclear area was modified to a rectangle $0.60 \times 2.00 \mathrm{~mm}$ in size, which corresponds to a $3 \times 10$ matrix of the measuring areas.

\section{Precision of the Measurements}

The three main random sources of variation in these measurements are variation among individuals, variation among photographs and variation among measurements (Table I).

The considerable variation in level of back scattering among individuals $\left(\sigma_{A}^{2}\right)$ and the small variation among

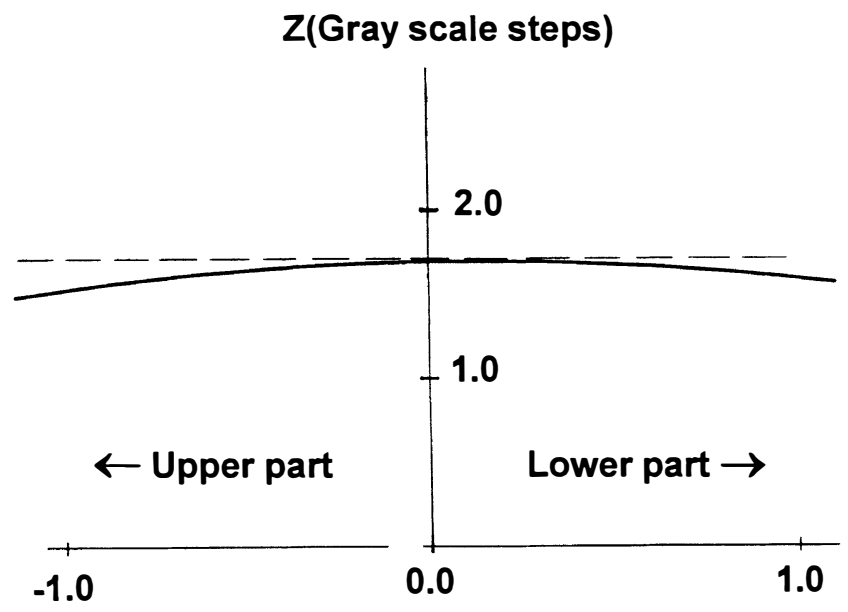

Distance from the center of the lens

Fig. 2. The spatial distribution of back scattering in the nucleus along an axis perpendicular to the anatomical axis of the lens in non-cataractous lenses, expressed as grey scale values $\left(\mathrm{Z}=1.683+0.041 \mathrm{X}-0.208 \mathrm{X}^{2}\right)$

photographs $\left(\sigma_{\mathrm{C}}^{2}\right)$ and measurements $\left(\sigma_{\varepsilon}^{2}\right)$ is consistent with our previous work. ${ }^{14}$ This indicates that one measurement in one photograph for each subject is enough for evaluation of distribution of back scattering in the nucleus in a population.

The precision of the measurement was estimated. As each area was measured only once, the measurement error was combined with the interaction among photographs, $X$-locations and $Y$-locations in the corresponding mean square (Table I: Int.(P-X-Y) and error). Considering the whole term as the measurement error, the precision of the measurement was about 3\%. Therefore, this precision should be a maximum value.

\section{Difference Between Sides}

No significant systematic difference between sides was found (Table I: Sides). Thus, it is not necessary to stratify sides when estimating the average nuclear back scattering in a population of non-cataractous lenses.

\section{Spatial Distribution of Back Scattering}

The finding that the interaction between the two dimensions (Table I: Int. $(\mathrm{X}-\mathrm{Y})$ ) was significant implies that the distribution of back scattering along the $X$-axis is different for different $Y$ layers. However, the significant interaction among individuals, $X$ - and $Y$-axis (Table I: Int.(I-X-Y)) indicates that there might be a large variation of the coeffi- 
cients among individuals for a surface polynomial. Thus, a large number of individuals would be needed in order to detect a change in nuclear opacity. Since the test statistic for the variation of spatial distribution along the $X$-axis was significant (Table I) while that for variation along the $Y$-axis was not significant (Table I), the spatial distribution of back scattering was simplified to one dimension. We average over $Y$-locations at each $X$-coordinate when establishing the polynomial.

In the present study we attempted to fit the spatial distribution of back scattering in non-cataractous lenses to a polynomial. The orthogonal comparisons (Table II) show that the variation in back scattering along the $X$-axis can be explained by a second-order polynomial $\left(Z=a_{0}+a_{1} X+a_{2} X^{2}\right)$. Therefore, a second-order polynomial was selected for further analysis. In this polynomial, $a_{0}$ reflects the level of the back scattering; $a_{1}$ and $a_{2}$ indicate the asymmetry and shape of the distribution curve, respectively.

Since nuclear cataract usually develops symmetrically around the anatomical axis of the lens, ${ }^{11}$ it is expected that an increase in the nuclear density could be reflected mainly by a change in the coefficients $a_{0}$ and $a_{2}$. The fact that the linear component $\left(a_{1}\right)$ in the polynomial is significant (Table II) indicates that the spatial distribution of back scattering is not symmetrical around the anatomical axis of the lens. The highest grey scale value is located slightly below the anatomical axis of the lens (Fig. 2). This asymmetry might be due to the gravity effect acting on the lens material. This finding indicates that the orientation of the image of the lens in Scheimpflug photograph is important.

The fact that the intensity of back scattering peaks in the central region and gradually decreases towards the periphery of the lens (Fig. 2) is consistent with the spatial distribution of protein concentration. ${ }^{19}$

\section{Sensitivity of the Method}

The physiological back scattering could be used as a basecurve in estimation of nuclear opacification. The alteration of back scattering in cataractous lenses could be determined as significant deviations from the base-curve values, measured as the regression coefficients.

Clinically, it was observed that nuclear cataract manifests symmetrically. ${ }^{11}$ Therefore we altered the original grey scale in five different patterns to estimate the influence of increased grey scale values on the regression coefficients of the polynomial. In Table $\mathrm{V}$, it can be seen that for a localised increase in the grey scale at the central region of the lens, $a_{2}$ is changed in the order of $100 \%$ of the mean, while $a_{0}$ is changed in the order of $10 \%$ of the mean. For the homogeneous increase in the grey scale level along the $X$-axis, the increase in grey scale level is proportional to the increase in the coefficients $a_{0}$ and $a_{2}$, respectively (Table V, pattern 5).

Asymmetrical increase in nuclear opacity might occur clinically. It is expected that the change in the coefficient $a_{1}$ could reflect this change. Further, tolerance limits of parameters for the physiological distribution of back scattering determined from a large sample could serve as reference values for individual measurements.

\section{Final Remarks}

In the present study the spatial distribution of back scattering in the nuclear area of the non-cataractous human lens was investigated. It is concluded that there is a characteristic variation in nuclear back scattering along a sagittal section of the nucleus centred on the pupil. This variation can be modelled as a second-order polynomial. Thus the regression coefficients of the polynomial can be used as cataract indices. We anticipate that the established method will improve the sensitivity in detection of nuclear cataract, especially in the early stages. It is believed that the increase in back scattering in the central region corresponds to the peak protein concentration in the centre of the nucleus.

\section{APPENDIXES}

\section{Appendix 1}

The experimental data were analysed with an analysis of variance for a mixed model: ${ }^{17}$

$$
\begin{aligned}
X_{i j k l m}= & \mu+A_{i}+\beta_{j}+C_{k(i j)}+\zeta_{l}+\eta_{m}+(A \beta)_{i j}+(\mathrm{A} \zeta)_{i l} \\
& \left.+(A \eta)_{i m}+(\beta \zeta)_{j l}+(\beta \eta)_{j m}+(\zeta \eta)_{l m}+(C \zeta)_{k l(i j)}\right) \\
& \left.+(\mathrm{C} \eta)_{k m i j}\right)+(A \beta \zeta)_{i j l}+(A \beta \zeta \eta)_{i j m}+(A \zeta \eta)_{i l m} \\
& +(\beta \zeta \eta)_{j l m}+(C \zeta \eta)_{k l m(i j)}+(A \beta \zeta \eta)_{i j l m}+\varepsilon_{(i j k l m)}
\end{aligned}
$$

Here, each grey scale value, $x_{i j k l m}$, equals the sum of the expected total mean, $\mu$; a term for the random variation among individuals, $A_{i}(i=1 \ldots 10)$; a term for the fixed effect of sides (right and left eye), $\beta_{j}(j=1 \ldots 2)$; a term for the random variation between photographs within side and individual, $C_{k(i j)}(k=1 \ldots 2)$; a term for the fixed effect of grey scale values along the $X$-axis ( $X$-locations), $\zeta_{l}(l=1 \ldots 10)$; a term for the fixed effect of grey scale values along the $Y$-axis ( $Y$-locations), $\eta_{m}(m=1 \ldots 3)$; a term for interaction between individuals and sides, $(A \beta)_{i j}$; a term for interaction between individuals and $X$-locations, $(A \zeta)_{i}$; a term for interaction between individuals and $Y$-locations, $(A \eta)_{i m}$; a term for interaction between sides and $X$-locations, $(\beta \zeta)_{j l}$; a term for interaction between sides and $Y$-locations, $(\beta \eta)_{j m}$; a term for interaction between $X$-locations and $Y$-locations, $(\zeta \eta)_{l m}$; a term for interaction between photographs and $X$-locations, $(C \zeta)_{k(i i j)}$; a term for interaction between photographs and $Y$-locations $(C \eta)_{k m(i j)}$; a term for interaction among individuals, sides and $X$-locations, $(A \beta \zeta)_{i j l}$; a term for interaction among individuals, sides and $Y$-locations, $(A \beta \eta)_{i j m}$; a term for interaction among individuals, $X$-locations and $Y$-locations, $(A \zeta \eta)_{i / m}$; a term for interaction among sides, $X$-locations and $Y$-locations, $(\beta \zeta \eta)_{j / m}$; a term for interaction among photographs, $X$-locations and $Y$-locations, $(C \zeta \eta)_{k \mid m(i j)}$; a term for interaction among individuals, sides, $X$-location and $Y$-locations, $(A \beta \zeta \eta)_{i j l m}$; and a term for random error of measurements, $\varepsilon_{(i j k l m)}$.

\section{Appendix 2}

(1) The regression coefficients $a_{0}, a_{1}$ and $a_{2}$ were each ana- 
lysed using an analysis of variance according to the following model:

$$
x_{i j k}=\mu+A_{i}+B_{j(i)}+C_{k(i j)}+\varepsilon_{(i j k)}
$$

Here, each value, $x_{i j k}$, equals the sum of the expected total mean, $\mu$; a term for the random variation among individuals, $A_{i}(i=1 \ldots a, a=10)$; a term for the random variation between sides, $B_{j(i)}(j=1 \ldots b, b=2)$; a term for the random variation between photographs within side and individual, $C_{k(i j)}(k=1 \ldots n, n=2)$; and a term for random error of measurements, $\varepsilon_{(i j k)}$.

(2) A 95\% confidence interval for $a_{0}, a_{1}$, and $a_{2}$, respectively, was estimated according to the following formulae:

$$
\begin{aligned}
& \mathrm{I}_{\mu}(95 \%)=\bar{x} \ldots \pm t_{9: 0.95} s(\bar{x} \ldots) \\
& s\left(\bar{x}_{\ldots}\right)=\sqrt{\frac{\text { mean square for individuals }}{a b n}}
\end{aligned}
$$

Here, $\bar{x} \ldots$ is the estimated total mean and $s\left(\bar{x}_{\ldots}\right)$ is the corresponding standard deviation. The mean square for individuals is obtained from the analysis of variance according to equation (3).

This study was supported by Wenner-Gren Center Foundation for Scientific Research, Karolinska Institutets forskningsfonder, Kronprinsessan Margaretas Arbetsnämd För Synskadade, Swedish Society of Medicine, Gun och Bertil Stohnes Stiftelse, Synfrämjandet forskningsfonder, Carmen och Bertil Regners Fond för forskning inom Området Ögonsjukdomar, Medical Research Council (project no. 4204) and Merck Sharp \& Dohme.

Key words: Lens nucleus, Photography, Photometry, Scattering, Scheimpflug photography.

\section{REFERENCES}

1. Datiles MB, Edwards PA, Trus BL, Green SB. In vivo studies on cataracts using the Scheimpflug slit lamp camera. Invest Ophthalmol Vis Sci 1987;28:1707-10.

2. Hockwin O, Dragomirescu V, Laser H. Measurements of lens transparency or its disturbances by densitometric image analysis of Scheimpflug photographs. Graefes Arch Clin Exp Ophthalmol 1982;219:255-62.

3. Hockwin O, Lerman S, Ohrloff C. Investigations on lens transparency and its disturbances by microdensitometric analyses of Scheimpflug photographs. Curr Eye Res 1984;3:15-22.
4. Hockwin O, Laser H, Kapper K. Image analysis of Scheimpflug negatives. Ophthalmic Res 1988;20:99-105.

5. Kashiwagi T, Khu PM. New method of measuring nuclear cataract in colour Scheimpflug photographs. Ophthalmic Res 1990;22 (Suppl 1):24-8.

6. Khu PM, Kashiwagi T. Quantitating nuclear opacification in colour Scheimpflug photographs. Invest Ophthalmol Vis Sci 1993;34:130-6.

7. Mayer H, Irion KM. New approach to area image analysis of Scheimpflug photos of the anterior eye segment. Ophthalmic Res 1985;17:106-10.

8. Shun-Shin GA, Bron AJ, Brown NP, Sparrow JM. The relationship between central nuclear scatter and perinuclear retrodots in the human crystalline lens. Eye 1992;6:407-10.

9. Sparrow JM, Brown NAP, Shun-Shin GA, Bron AJ. The Oxford modular cataract image analysis system. Eye 1990;4:638-48.

10. Harris ML, Smith GTH, Brown NAP. Inter- and intra-observer reproducibility of the new Oxford CCD Scheimpflug camera. Eye 1991;5:487-90.

11. Chylack LT Jr, McCarthy D, Khu P. Use of Topcon SL-45 Scheimpflug slit photography to measure longitudinal growth of nuclear cataracts in vivo. Lens Res 1988;5:83-93.

12. Chylack LT, Rosner B, Cheng HM, McCarthy D, Pennett M. Sources of variance in the objective documentation of human cataractous change with Topcon SL-45 and NeitzCRT retroillumination photography and computerized image analysis. Curr Eye Res 1987;6:1381-9.

13. Chen SY, Chylack LT Jr, White O. Topcon SL-45 photography, a suitable technique for documenting nuclear but not cortical cataractous change in vivo. Invest Ophthalmol Vis Sci(ARVO Suppl) 1985;26:119.

14. Qian W, Söderberg PG, Chen E, Magnius K, Philipson B. A common lens nuclear area in Scheimpflug photographs. Eye 1993;7:799-804.

15. Lindström B, Philipson B. Microdensitometer system for microradiography. Histochemie 1969;17:187-93.

16. Qian W, Söderberg PG, Chen E, Magnius K, Philipson B. Evaluation of grey scale steps on Scheimpflug photographs. Acta Ophthalmol (Copenh) 1993;71:79-84.

17. Snedecor GW, Cochran WG. The random effects model. In: Statistical methods, 7th ed. Ames, Iowa: Iowa State University Press, 1980:238-54.

18. Freund RJ, Minton PD. Orthogonal polynomials. In: Regression methods. New York: Dekker, 1979:162-71.

19. Philipson B. Distribution of protein within the normal rat lens. Invest Ophthalmol Vis Sci 1969;8:258-70.

20. Snedecor GW, Cochran WG. The comparison of two samples. In: Statistical methods, 7 th ed. Ames, Iowa: Iowa State University Press, 1980:102-5. 